\title{
Botrychium schaffneri Underw. extract acts via DIABLO to induce apoptosis and inhibit proliferation of non-small cell lung carcinoma in vitro and in vivo
}

\author{
Yu-Jia Liu ${ }^{1 \#, ~ Y i-W e n ~ Z h a n g ~}{ }^{1,2 \#}$, Shui-Lian Zheng', Xiao-Chun Zheng', Hai-Ying Ding ${ }^{3}$, Wen-Xiu Xin ${ }^{3}$, \\ Jiao Sun ${ }^{3}, \mathrm{Li} \mathrm{Li}^{4}$, Ping Huang ${ }^{1,2}$ \\ ${ }^{1}$ Clinical Pharmacy Center, Department of Pharmacy, Zhejiang Provincial People's Hospital; Affiliated People's Hospital, Hangzhou Medical \\ College, Hangzhou, China; ${ }^{2}$ Key Laboratory of Endocrine Gland Diseases of Zhejiang Province, Hangzhou, China; ${ }^{3}$ Department of Pharmacy, \\ Institute of Cancer Research and Basic Medical Sciences of Chinese Academy of Sciences, Cancer Hospital of University of Chinese Academy of \\ Sciences, Zhejiang Cancer Hospital, Hangzhou, China; ${ }^{4}$ Department of Pharmacy, The First People's Hospital of Chun An, Zhejiang Hangzhou, \\ China \\ Contributions: (I) Conception and design: YJ Liu, P Huang; (II) Administrative support: P Huang; (III) Provision of study materials or patients: YW \\ Zhang, L Li; (IV) Collection and assembly of data: SL Zheng, XC Zheng; (V) Data analysis and interpretation: HY Ding, WX Xin, J Sun, L Li; (VI) \\ Manuscript writing: All authors; (VII) Final approval of manuscript: All authors. \\ \#These authors contributed equally to this work. \\ Correspondence to: Ping Huang. Clinical Pharmacy Center, Department of Pharmacy, Zhejiang Provincial People's Hospital; Affiliated People's \\ Hospital, Hangzhou Medical College, 158 Shangtang Road, Hangzhou 310014, China. Email: huangpwly@sina.com.
}

Background: Botrychium schaffneri Underw. has been popularly consumed since ancient times as a traditional medicine in China to treat whooping cough, bronchial asthma, and febrile convulsive twitch disease. This led us to investigate whether Botrychium schaffneri Underw. extract (BSE) may be effective against lung cancer, especially non-small cell lung carcinoma (NSCLC).

Methods: In this study, we extracted the ethanolic root extract of the grass, Botrychium schaffneri Underw. In vitro study, the change of NCI-H1299 cell proliferation was observed with CCK8 and MTT assays. Cell apoptosis was assessed using a kit based on staining with FITC-conjugated annexin V. In vivo study, we establish a stable animal model of NSCLC in nude mice, tumor volume and weight was measured twice a week. We conduct gene microarray screened for differentially expressed genes (DEGs), between NCI-H1299 cells treated by BSE or not. Then the DEGs were functionally annotated and path enriched.

Results: It was revealed that BSE significantly suppressed NSCLC cell proliferation ( $\mathrm{IC}_{50} 134 \mu \mathrm{g} / \mathrm{mL}$ ) and induced apoptosis. It also slowed tumor growth without affecting body weight, and a dose of $25 \mathrm{~g} / \mathrm{kg}$ led to significantly smaller tumors than in control animals (13.85 \pm 3.36 vs. $23.40 \pm 6.05, \mathrm{P}=0.044)$. Apoptosisrelated protein direct IAP Binding protein with low PI (DIABLO) expression was up-regulated by BSE, and DIABLO knockdown significantly attenuated the anti-tumor effects of the extract.

Conclusions: In conclusion, BSE reduces the viability of NSCLC cells and promotes apoptosis, and these effects may be mediated by DIABLO.

Keywords: Botrychium schaffneri Underw. extract (BSE); non-small cell lung carcinoma (NSCLC); apoptosis; direct IAP Binding protein with low PI (DIABLO)

Submitted Sep 17, 2021. Accepted for publication Nov 17, 2021.

doi: 10.21037/atm-21-5409

View this article at: https://dx.doi.org/10.21037/atm-21-5409 


\section{Introduction}

Lung cancer is a major cause of cancer-related deaths worldwide. Non-small cell lung carcinoma (NSCLC) accounts for approximately $80 \%$ of lung cancer diagnoses and is associated with a 5 -year survival rate of only $11 \%$ (1). Platinum-based chemotherapy and radiotherapy are widely used treatments, but NSCLC can develop resistance to both, leading to relapse when the primary tumor becomes metastatic $(2,3)$. Therefore, a novel, efficient, and lowtoxicity therapy for NSCLC is urgently needed.

An ethanolic extract of the entire root of the grass Botrychium schaffneri Underw. (BSE) has been used in China for centuries to treat whooping cough, bronchial asthma, and febrile convulsive twitch disease $(4,5)$. More recently, BSE has been shown to have expectorant and antiinflammatory effects. It has also been shown that BSE and its components exert anti-inflammatory and anti-allergic effects in mice, as well as inhibit the proliferation of skin papilloma (6,7). In our previous work, we showed that BSE may be effective against severe asthma (8). This led us to question whether BSE may be effective against lung cancer, in particular NSCLC.

In this study, we examined whether BSE exerted antitumor effects over NSCLC cell lines in vitro and over NSCLC xenografts in mice. We also examined whether anti-tumor effects of the extract involved altering the expression of DIABLO, also known as the "second mitochondria-derived activator of caspases" (Smac). The DIABLO protein is released from mitochondria and reactivates caspases that have been suppressed by inhibitors of apoptosis proteins (IAP) $(9,10)$, and DIABLO downregulation has been linked to cancer progression. These results suggest that DIABLO may help protect against cancer. Consistent with this, the Smac mimetic JP1201 sensitizes NSCLC to combination chemotherapy (11).

We present the following article in accordance with the ARRIVE reporting checklist (available at https://dx.doi. org/10.21037/atm-21-5409).

\section{Methods}

\section{Cell culture and transfection}

The human NSCLC cell line NCI-H1299 (CellCook Biotech, Guangzhou, China) was cultured in Roswell Park Memorial Institute (RPMI)-1640 medium (Life Technologies, Carlsbad, CA, USA) containing 10\% heat-inactivated fetal bovine serum (FBS; GE Healthcare Life Sciences Hyclone
Laboratories, Chicago, IL, USA), penicillin (100 units/mL), and streptomycin $(100 \mathrm{mg} / \mathrm{mL})$ at $37{ }^{\circ} \mathrm{C}$ in $5 \% \mathrm{CO}_{2}$. A day before transfection, cells were seeded into 6-well plates, cultured overnight, then transfected with a short interfering RNA against DIABLO (si-DIABLO) or a negative control RNA (si-NC) (Biomics Biotechnologies, Jiangsu, China). Transfections were performed for $6 \mathrm{~h}$ using Lipofectamine ${ }^{\circledR}$ 3000 (Invitrogen, Thermo Fisher Scientific, Waltham, MA, USA), according to the manufacturer's protocol. We chose 3 different si-DIABLO sequences (Table 1); si-DIABLO-3 showed the highest efficiency and was chosen for subsequent assays (Figure S1). After transfection, the medium was replaced with fresh RPMI-1640 containing 10\% FBS and antibiotics, as described above.

\section{Botrychium schaffneri Underw. extract}

In this study, we used the ethanolic root extract of the grass, Botrychium schaffneri Underw., which is known as Sceptridium ternatum, Yin Di Jue, or Xiao Chun Hua. Botrychium schaffneri Underw. was collected from Lishui in the Zhejiang Province, China $\left(118^{\circ} 41^{\prime} \mathrm{E}-120^{\circ} 26^{\prime} \mathrm{E}, 27^{\circ} 25^{\prime} \mathrm{N}-28^{\circ} 57^{\prime} \mathrm{N}\right.$; voucher specimen ZJ-20120926), and the species was confirmed by Professor Xi-Lin Chen (Zhejiang Chinese Medicine University) to meet the specifications in the Chinese Pharmacopoeia. Botrychium schaffneri Underw. $(10 \mathrm{~kg})$ was mixed with 10 volumes of $70 \%(\mathrm{v} / \mathrm{v})$ aqueous ethanol and subjected to reflux extraction under heat. A total of 3 cycles of extraction were performed for 2 hours each time. The filtrates were combined and concentrated under reduced pressure, and the resulting ethanol extract was diluted in water to a density of $2 \mathrm{~g} / \mathrm{mL}$, representing the crude drug extract.

\section{Cell viability assay}

Cells were seeded into 96-well plates, cultured overnight, then treated for $72 \mathrm{~h}$ with different doses of BSE. The cell viability was determined using Cell Counting Kit- 8 and MTT assay. CCK-8 solution $(10 \mu \mathrm{L})$ was added to each well, and the plates were incubated for $1 \mathrm{~h}$ at room temperature; MTT solution $(20 \mu \mathrm{L})$ was added to each well and incubated for a further $4 \mathrm{~h}$ at $37^{\circ} \mathrm{C}$, then aspirated and $100 \mu \mathrm{L}$ dimethyl sulfoxide (DMSO) was added to dissolve the formazan crystals. Cell survival was quantified in terms of the number of viable cells by measuring absorbance at $450 \mathrm{~nm}$ using a microplate reader (BioTek Instruments, Winooski, VT, USA). Average $\mathrm{IC}_{50}$ values were calculated from at least 
Table 1 Short interfering RNA primers

\begin{tabular}{ll}
\hline Target gene & Sequence \\
\hline si-DIABLO-1 & Forward 5'-CCGACAAUAUACAAGUUUAdTdT-3' \\
& Reverse 5'-UAAACUUGUAUAUUGUCGGdTdT-3' \\
si-DIABLO-2 & Forward 5'-GGCAGAAGCACAGAUAGAAdTdT-3' \\
& Reverse 5'-UUCUAUCUGUGCUUCUGCCdTdT-3' \\
si-DIABLO-3 & Forward 5'-CCUAUUACUACUAAGAGAAdTdT-3' \\
& Reverse 5'-UUCUCUUAGUAGUAAUAGGdTdT-3' \\
Negative & Forward 5'-UUCUCCGAACGUGUCACGUdTdT-3' \\
control & Reverse 5'-ACGUGACACGUUCGGAGAAdTdT-3'
\end{tabular}

DIABLO, direct IAP Binding protein with low PI.

Table 2 RT-PCR primers

\begin{tabular}{ll}
\hline Target gene & Sequence \\
\hline DIABLO & Forward 5'-CCTTCGTGCTCCTCAGCTAC-3' \\
& Reverse 5'-AGGAAGTAAGTGGTCCAGCC-3' \\
GAPDH & Forward 5'-GTCATCCATGACAACTTTGG-3' \\
& Reverse 5'-GAGCTTGACAAAGTGGTCGT-3' \\
\hline
\end{tabular}

RT-PCR, real-time PCR; DIABLO, direct IAP Binding protein with low PI; GAPDH, glyceraldehyde-3-phosphate dehydrogenase.

3 independent experiments using the "log it" method (12).

\section{Apoptosis assay}

Cells were cultured overnight in 6-well plates and treated for $24 \mathrm{~h}$ with BSE. Apoptosis in the cultures was assessed using a kit based on staining with FITC-conjugated annexin V (catalog no. C1062S, Beyotime, Shanghai, China) and flow cytometry (Beckman Coulter, Brea, CA, USA). Cells were suspended in a mixture of $1 \mathrm{X}$ annexin V-FITC binding buffer $(195 \mu \mathrm{L})$ and annexin V-FITC $(5 \mu \mathrm{L})$, incubated at room temperature for $10 \mathrm{~min}$, and centrifuged at $1,000 \times \mathrm{g}$ for $5 \mathrm{~min}$. The pellet was resuspended in binding buffer $(190 \mu \mathrm{L})$, and propidium iodide (PI) working solution $(10 \mu \mathrm{L})$ was added before the samples were analyzed using flow cytometry.

\section{DAPI assay}

Cells were cultured overnight in 6-well plates, treated with BSE for $24 \mathrm{~h}$, washed twice with phosphate-buffered saline (PBS), then incubated for $30 \mathrm{~min}$ with 4',6-diamidino-2- phenylindole (DAPI, $1 \mathrm{mg} / \mathrm{mL}$ ). Stained nuclei were observed under a fluorescence microscope (DMI 4000 B, Leica, Wetzlar, Germany). Cells with nuclei showing fragmentation and chromatin condensation were considered apoptotic.

\section{Western blotting}

Protein extracts were resolved by $8-15 \%$ sodium dodecyl sulfate-polyacrylamide gel electrophoresis (SDS-PAGE) and transferred onto polyvinylidene difluoride (PVDF) membranes. The membranes were blocked with $5 \%$ bovine serum albumin (BSA) for $1 \mathrm{~h}$ and incubated with primary antibodies against the following proteins: intact poly (ADP-ribose) polymerase (PARP) or cleaved PARP (Santa Cruz Biotechnology, Santa Cruz, CA, USA), full-length or cleaved caspases-3 and -9 (Cell Signaling Technology, Danvers, MA, USA), or glyceraldehyde 3-phosphate dehydrogenase (GAPDH) or DIABLO (Proteintech, Rosemont, IL, USA). Membranes were then blotted with horseradish peroxidase (HRP)-conjugated secondary antibodies (Jackson Immuno Research Laboratories, West Grove, PA, USA), followed by enhanced chemiluminescence detection (Biological Industries, Beit HaEmek, Israel).

\section{Quantitative real-time polymerase chain reaction}

Quantitative real-time polymerase chain reaction (qRTPCR) was performed to measure levels of DIABLO messenger RNA (mRNA). Total RNA was extracted and reverse-transcribed into complementary DNA (cDNA) using SuperScript (Thermo Fisher, Waltham, MA, USA). The DIABLO mRNA levels were quantified relative to levels of GAPDH mRNA using SYBR Green (Thermo Fisher, USA) and the $2^{-\triangle \triangle C T}$ method. The primers used in this assay are shown in Table 2.

\section{Animal experiments}

A total of 20 female BALB/c nude mice (4-6 weeks old) were purchased (Shanghai Sippr-BK Laboratory Animals, Shanghai, China) and allocated to 4 groups ( 5 mice per group). The NCI-H1299 cells were injected subcutaneously into the left side of the posterior flank. Once tumor volumes reached about $100 \mathrm{~mm}^{3}$, control group mice were treated with $0.9 \%$ normal saline and the others were treated with a different dose of BSE $(1,5$, or $25 \mathrm{~g} / \mathrm{kg}$ ), injected into the caudal vein. Tumor volume was measured twice a week and mice were euthanized at 25 days after implantation. Animal 
experiments were approved by the Animal Experimental Ethics Committee of the Center for Drug Safety Evaluation and Research of Zhejiang University (ZJU) (No. IACUC-17-163) and were in compliance with the guidelines of Institution Animal Care and Use Committee of Center for Drug Safety Evaluation and Research (CDSER) of Zhejiang University (ZJU) for the care and use of animals. A protocol was prepared before the study without registration.

\section{Gene microarray analysis}

Total RNA was extracted using TRIZOL Reagent (Cat\#15596-018, Life Technologies, Carlsbad, CA, USA) following the manufacturer's instructions and checked to inspect RNA integrity. Qualified total RNA was further purified by RNeasy mini kit (Cat\#74106, QIAGEN, $\mathrm{GmBH}$, Hilden, Germany) and labeled. Each slide was hybridized with $600 \mathrm{ng}$ Cy3-labeled cRNA using Gene Expression Hybridization Kit (Cat\#5188-5242, Agilent Technologies, Santa Clara, CA, US), then slides were scanned with default settings. The original data from the gene microarray were adjusted using the limma package (13) and screened for differentially expressed genes (DEGs), defined as genes whose expression differed between NCI-H1299 cells which treated by BSE or not by $2^{\mid \log 2 \text { (fold change) }}>1$ with $\mathrm{P}<0.05$. The database for annotation, visualization, and integrated discovery (DAVID 6.8; https:// david.ncifcrf.gov/) was used to analyze DEGs for Gene Ontology (GO) (14). We used the Kyoto Encyclopedia of Genes and Genomes (KEGG) knowledge database to identify enrichment of biochemical pathways among DEGs, and R Profiler (3.6.0) was used to annotate and visualize the KEGG pathways of DEGs (15). We selected GO terms and pathways enriched by DEGs based on a cut-off criterion of $\mathrm{P}<0.05$.

\section{Gene set enrichment analysis (GSEA)}

GSEA (https://software.broadinstitute.org/gsea/index. jsp) was used to assess DIABLO related pathways and molecular mechanisms in NSCLC samples (16). Enriched gene sets with $\mathrm{P}$ value of $<0.05$ were considered statistically significant.

\section{Statistical analysis}

All experiments were performed in at least three biological replicates. Data were presented as mean \pm SD. Inter-group differences were assessed for significance using the 2-tailed Student's $t$-test.

\section{Results}

BSE suppressed proliferation and promoted apoptosis in NSCLC cell cultures

It was shown that BSE significantly inhibited proliferation of NCI-H1299 cells in a dose- and time-dependent manner by CCK8 and MTT assays (Figure 1A,1B). It also induced apoptosis (Figure 1C,1D), which was associated with increases in levels of the cleaved forms of PARP and caspases-3 and -9 (Figure 1E). Moreover, when treated with Caspase inhibitor Z-VAD-FMK, the percentage of cell apoptosis was decreased as shown in Figure 1D. All of these results suggested that BSE induced apoptosis in NCI-H1299 cells mediated via caspase signal pathway.

\section{BSE suppressed growth of NCI-H1299 xenografts in nude mice}

Without exerting obvious toxicity on xenograft-bearing mice (Figure $2 A$ ), BSE reduced tumor volume relative to control tumors in a dose-dependent manner (Figure $2 B$ ). At 25 days after treatment, relative tumor volume was significantly smaller in animals treated with $25 \mathrm{~g} / \mathrm{kg}$ BSE $(13.85 \pm 3.36)$ than in control animals $(23.40 \pm 6.05, \mathrm{P}=0.044$; Figure $2 C$ ). A corresponding dose-dependent decrease in tumor weight was also observed (Figure 2D,2E).

\section{BSE promoted apoptosis of NSCLC cells in vivo}

The above anti-tumor effects correlated with an increase in apoptosis based on immunostaining of tumor sections for cleaved forms of PARP and caspase-3 (Figure 3A,3B). In fact, BSE doubled the proportion of tumor cells containing cleaved PARP, from $20 \%$ to $40 \%$.

\section{Identification of DEGs as a result of BSE treatment}

To identify other proteins that may help mediate the anti-tumor effects of BSE on NSCLC, we profiled the transcriptomes of NCI-H1299 cells in cultures exposed to BSE $(100 \mu \mathrm{g} / \mathrm{mL})$ and compared to these cultures undergoing no treatment. After applying stringent cut-offs, we were able to identify 10 up-regulated genes (SNX29, 

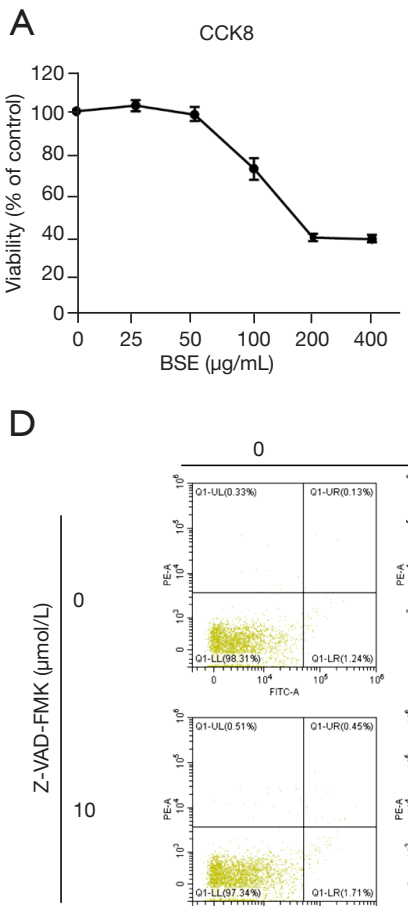

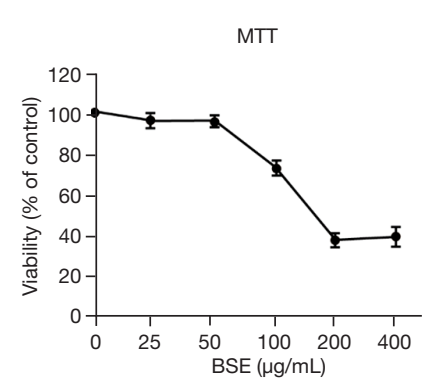

BSE $(\mu \mathrm{g} / \mathrm{mL})$

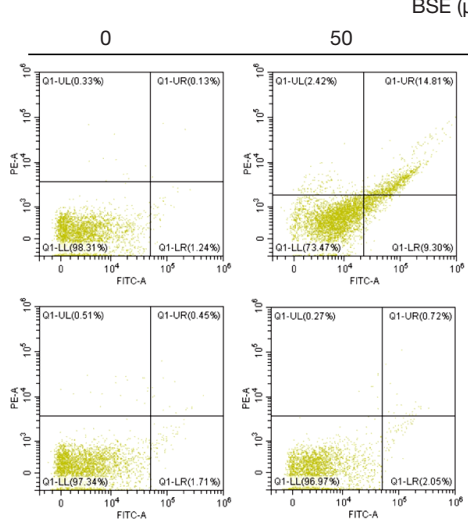

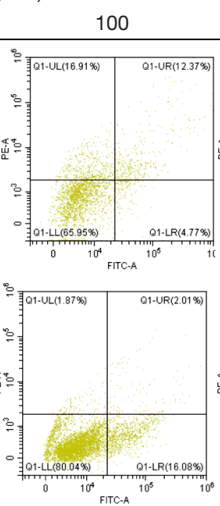

B



C $\quad 0^{B S E}(\mu \mathrm{g} / \mathrm{mL})$

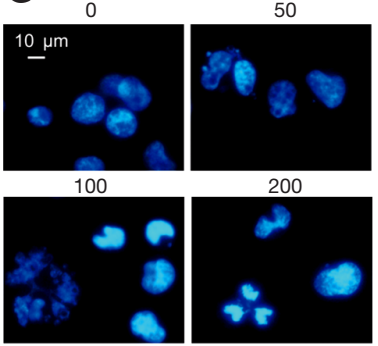

$E$
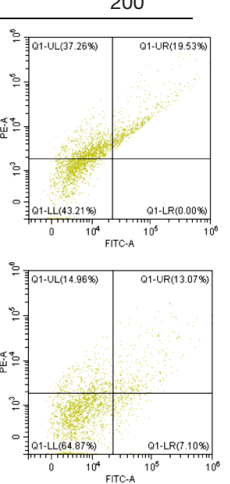



Figure 1 Effects of BSE on proliferation and apoptosis of the NSCLC cell line NCI-H1299 in vitro. (A) Viability of BSE-treated cells based on the CCK-8 assay (left), MTT assay (right); (B) change in cell numbers; (C) fluorescence microscopy of DAPI-stained cells after treatment with BSE; (D) flow cytometry of cells after treatment with BSE, Z-VAD-FMK and staining with annexin V/PI. The numbers in parentheses refer to the percentages of the total population falling within the gating; (E) Western blotting of apoptosis-related proteins in cells following treatment with BSE. BSE, Botrychium schaffneri Underw. extract; NSCLC, non-small cell lung cancer; CCK-8, Cell Counting Kit-8; MTT, Methyl Thiazolyl Tetrazolium; Z-VAD-FMK, Caspase inhibitor; DAPI, 4',6-diamidino-2-phenylindole; PI, propidium iodide.
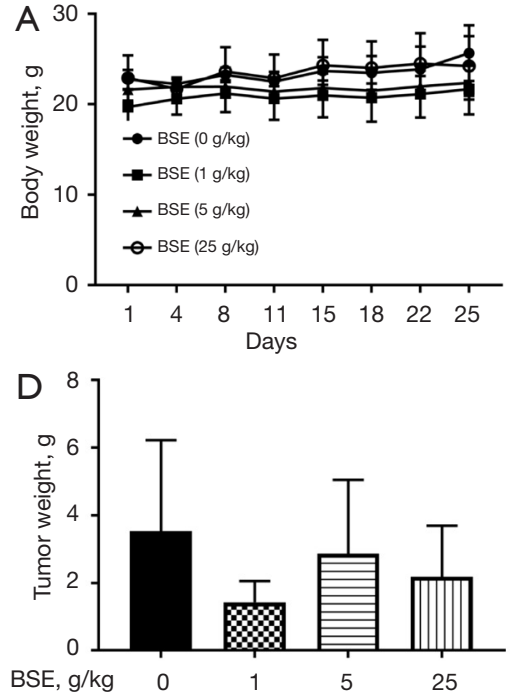

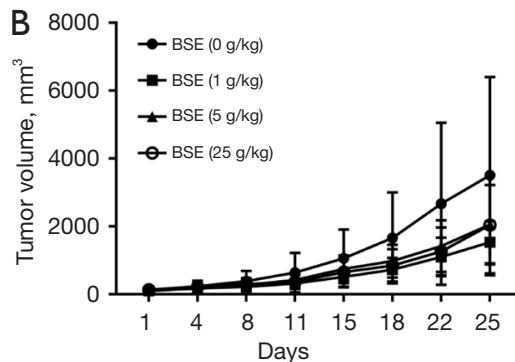

$E$

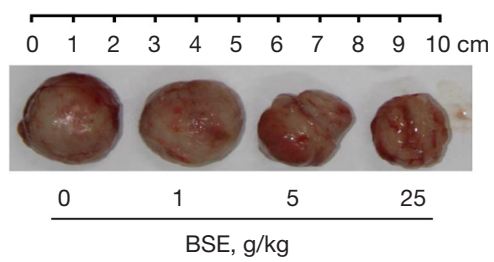

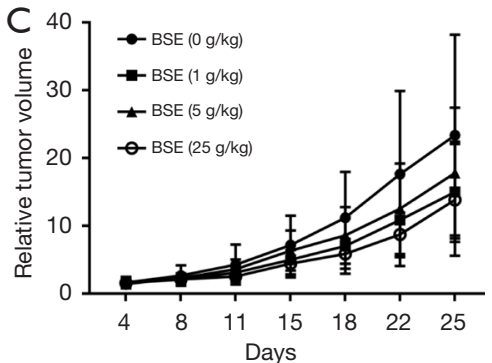

Days

Figure 2 Assessment of anti-tumor effects of BSE in nude mice with NCI-H1299 xenografts measured up to 25 days after treatment with extract. (A) Body weight; (B) tumor volume; (C) relative tumor volume; (D) tumor weight; (E) representative photographs of tumors. BSE, Botrychium schaffneri Underw. extract. 


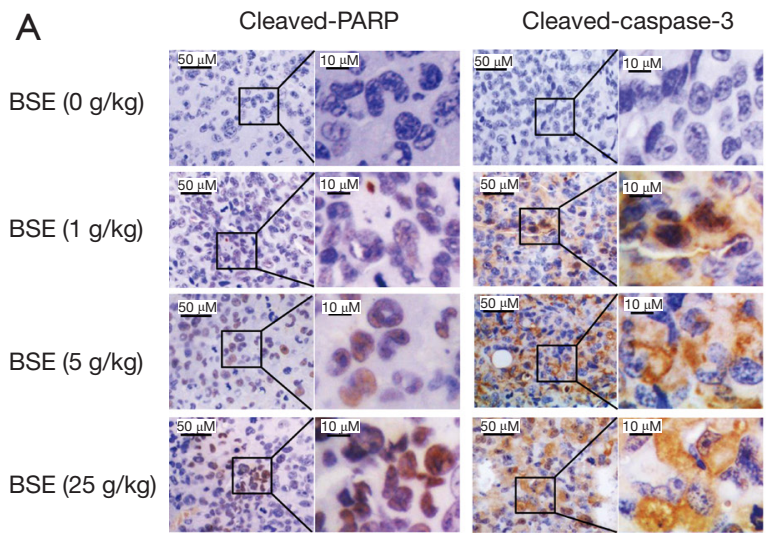

\begin{tabular}{|c|c|c|c|c|c|c|c|c|}
\hline \multirow{2}{*}{ B } & \multicolumn{4}{|c|}{ Cleaved-PARP } & \multicolumn{4}{|c|}{ Cleaved-caspase-3 } \\
\hline & - & + & ++ & +++ & - & + & ++ & +++ \\
\hline BSE (0 g/kg) & 4 & 1 & 0 & 0 & 1 & 3 & 1 & 0 \\
\hline BSE (1 g/kg) & 3 & 1 & 1 & 0 & 0 & 1 & 3 & 1 \\
\hline BSE (5 g/kg) & 3 & 1 & 1 & 0 & 0 & 0 & 4 & 1 \\
\hline BSE $(25 \mathrm{~g} / \mathrm{kg})$ & 3 & 1 & 1 & 0 & 0 & 2 & 3 & 0 \\
\hline
\end{tabular}

Figure 3 Apoptosis-related protein expression in NCI-H1299 xenografts in mice after treatment with BSE. Tumor sections were stained with hematoxylin-eosin, then with antibodies against the cleaved forms of PARP and caspase-3. (A) Reactivity assessment in tissue slices; (B) semi-quantification of cleaved forms of PARP and caspase-3 immunostaining intensity. The intensity was scored as follows: -, negative; +, weak; ++, moderate; +++, strong. BSE, Botrychium schaffneri Underw. extract; PARP, poly (ADP-ribose) polymerase.

Table 3 Differential expression of genes in NCI-H1299 cells after treatment with BSE $(100 \mu \mathrm{g} / \mathrm{mL})$

\begin{tabular}{lcc}
\hline Gene & Fold change & P value \\
\hline SNX29 & 4.25 & 0.01 \\
DIABLO & 4.05 & 0.04 \\
GPR152 & 3.93 & 0.01 \\
MTRNR2L2 & 3.79 & 0.00 \\
HLA-J & 3.25 & 0.03 \\
SHISA7 & 3.20 & 0.01 \\
KCP & 3.05 & 0.01 \\
AZGP1 & 2.49 & 0.05 \\
C6orf99 & 2.45 & 0.05 \\
LRRC39 & 2.06 & 0.02 \\
ZNF546 & 0.50 & 0.01 \\
ANK2 & 0.48 & 0.02 \\
FAM186B & 0.46 & 0.02 \\
B4GALT6 & 0.46 & 0.04 \\
FBXW12 & 0.42 & 0.03 \\
\hline BSE, BotyChim & &
\end{tabular}

BSE, Botrychium schaffneri Underw. extract; SNX29, Sorting Nexin 29; DIABLO, direct IAP Binding protein with low PI; GPR152, G Protein-Coupled Receptor 152; MTRNR2L2, MTRNR2 Like 2; HLA-J, Major Histocompatibility Complex, Class I, J (Pseudogene); SHISA7, Shisa Family Member 7; KCP, Kielin Cysteine Rich BMP Regulator; AZGP1, Alpha-2-Glycoprotein 1, Zinc-Binding; C6orf99, Chromosome 6 Putative Open Reading Frame 99; LRRC39, Leucine Rich Repeat Containing 39; ZNF546, Zinc Finger Protein 546; ANK2, Ankyrin 2; FAM186B, Family With Sequence Similarity 186 Member B; B4GALT6, Beta-1,4-Galactosyltransferase 6; FBXW12, F-Box And WD Repeat Domain Containing 12.
DIABLO, GPR152, MTRNR2L2, HLA-7, SHISA7, KCP, $A Z G P 1$, C6orf99, LRRC39) and 5 down-regulated genes (ZNF546, ANK2, FAM186B, B4GALT6, FBXW12) (Table 3). The GO analysis showed that these DEGs were significantly enriched in 'integral' and 'intrinsic' components of the plasma membrane, and the most important genes were DIABLO, GPR152, ANK2, and SHISA7 (Figure 4A,4B). The KEGG pathway analysis indicated that DEGs were significantly enriched in 'sphingolipid metabolism' (key gene: B4GALT6) and 'apoptosis' (key gene: DIABLO) (Figure $4 C$ ). GSEA shows that high DIABLO expression group were mainly enriched in GO biological process related to cell apoptosis (Figure $4 D$ ).

\section{Role of DIABLO in the anti-tumor effects of BSE on NSCLC cells}

In an effort to elucidate the molecular pathways by which BSE exerts anti-tumor effects, we investigated the genes of which the expression was altered by BSE treatment. We began by examining DIABLO, which has been previously linked to cancer progression. We found that BSE significantly up-regulated DIABLO mRNA levels in NCI-H1299 cultures (Figure 5A) and xenografts in mice (Figure $5 B$ ).

To obtain further direct evidence showing that DIABLO may help mediate the effects of the BSE, we knocked down its expression using siRNA. This knockdown substantially weakened the extract's anti-tumor effects (Figure 5C,5D) and its ability to up-regulate apoptosis-related proteins 


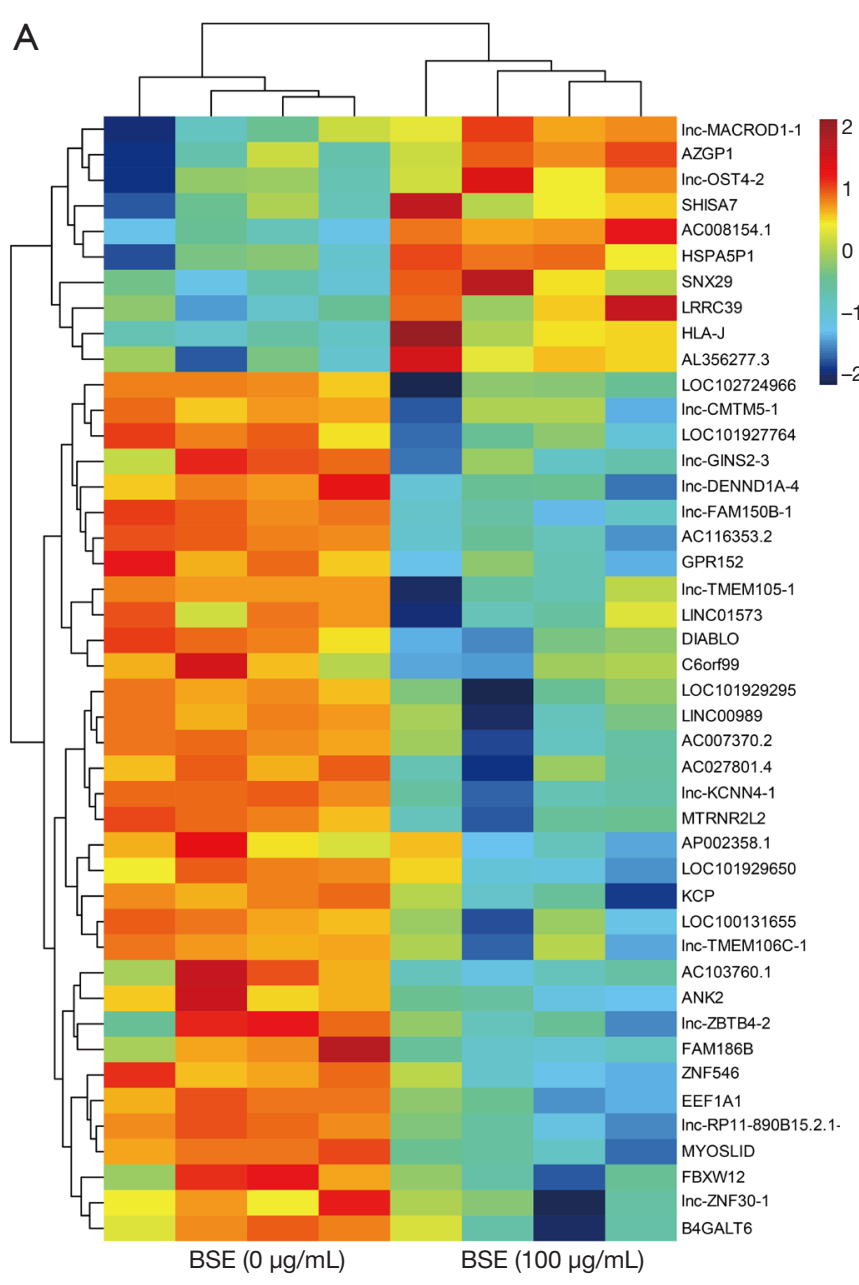

B

GO enrichment

\begin{tabular}{lccc}
\hline Description & Q value & Count & Genes \\
\hline $\begin{array}{l}\text { Integral component of } \\
\text { plasma membrane }\end{array}$ & 0.01656 & 4 & $\begin{array}{l}\text { DIABLO, GPR152, } \\
\text { ANK2, SHISA7 }\end{array}$ \\
$\begin{array}{l}\text { Intrinsic component of } \\
\text { plasma membrane }\end{array}$ & 0.01808 & 4 & $\begin{array}{l}\text { DIABLO, GPR152, } \\
\text { ANK2, SHISA7 }\end{array}$ \\
\hline C & KEGG enrichment & \\
\hline Description & Q value & Count & Genes \\
\hline $\begin{array}{l}\text { Sphingolipid } \\
\text { metabolism }\end{array}$ & 0.001224 & 1 & B4GALT6 \\
Apoptosis & 0.005181 & 1 & DIABLO \\
\hline
\end{tabular}
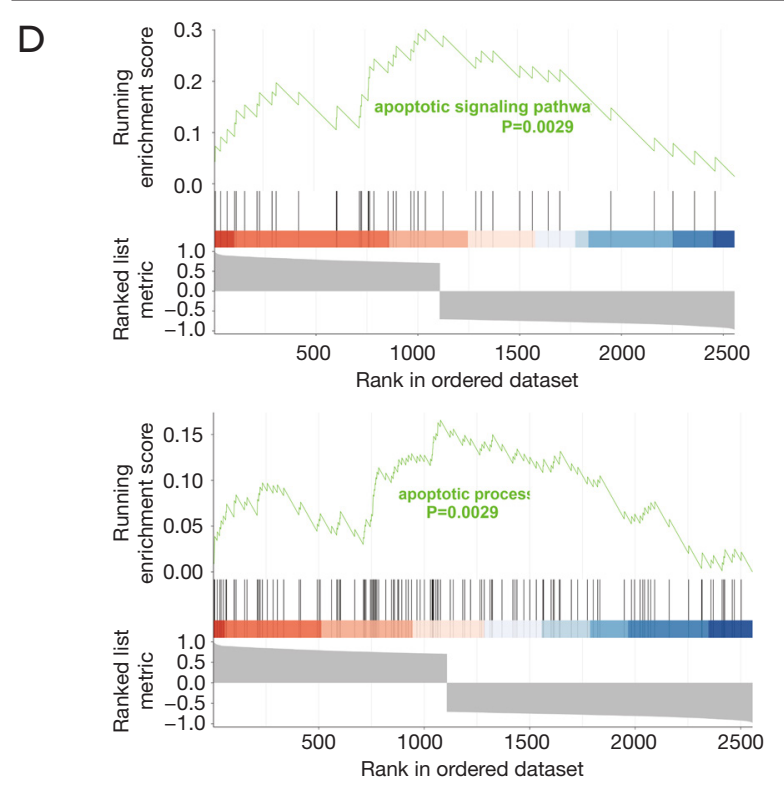

Figure 4 Differential expressions of genes in NCI-H1299 cells after treatment with BSE. (A) Cluster heat map of differentially expressed genes; (B) GO enrichment analysis of DEGs; (C) analysis of KEGG pathway enrichment among DEGs; (D) GSEA showing high-DIABLO groups are associated with cell apoptosis-related pathways; BSE, Botrychium schaffneri Underw. extract; GO, Gene Ontology; KEGG, Kyoto Encyclopedia of Genes and Genomes; DEGs, differentially expressed genes; GSEA, gene set enrichment analysis.

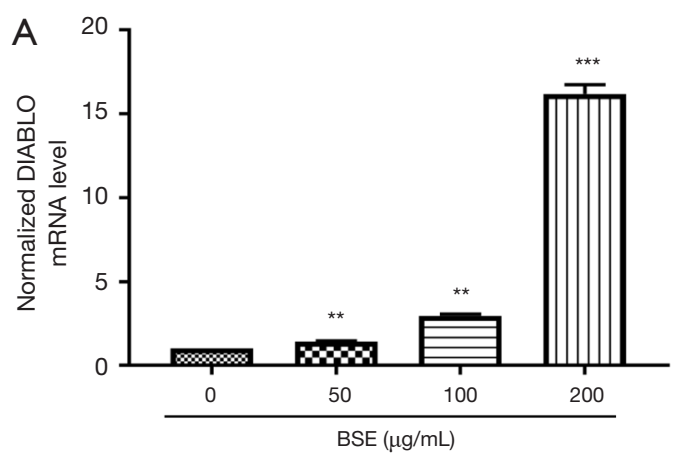

B

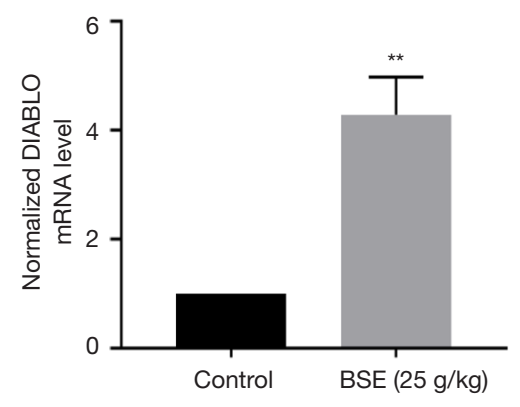



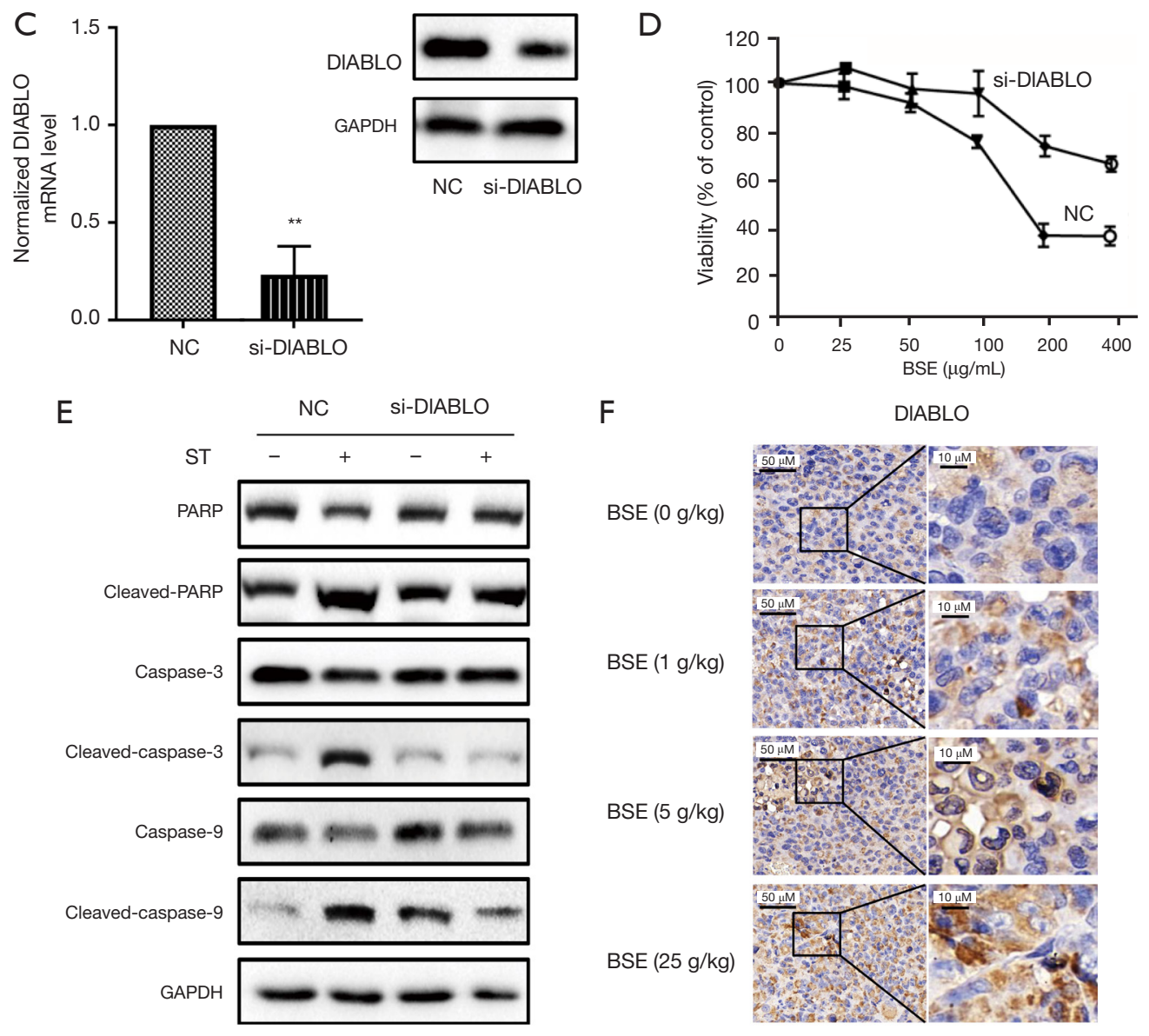

F

DIABLO

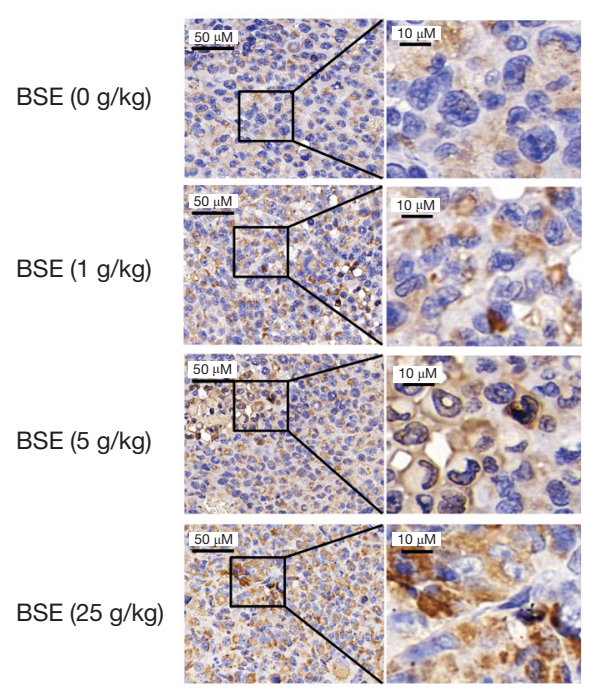

G

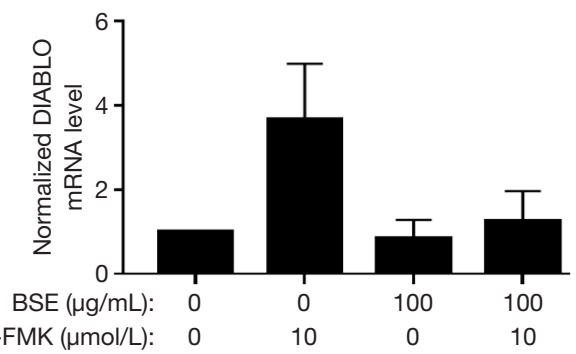

Figure 5 Effects of BSE on DIABLO expression in NCI-H1299 cells in vitro and in vivo. (A) DIABLO mRNA levels in cultures after treatment; (B) DIABLO mRNA levels in xenograft tumors in mice after treatment; (C) DIABLO levels in NCI-H1299 cultures after transfection with siRNAs against DIABLO (si-DIABLO) or negative control siRNA (si-NC); (D) proliferation of NCI-H1299 cells previously transfected with si-NC or si-DIABLO, followed by BSE; (E) Western blotting of apoptosis-related proteins in cells previously transfected with si-NC or si-DIABLO, followed by BSE (or not); (F) anti-DIABLO immunostaining of NCI-H1299 xenografts from mice treated with BSE (or not); (G) DIABLO levels in NCI-H1299 treated with BSE, Z-VAD-FMK. **, P<0.01; ***, P<0.001. BSE, Botrychium schaffneri Underw. extract; mRNA, messenger RNA; siRNAs, short interfering RNAs; DIABLO, direct IAP Binding protein with low PI; Z-VAD-FMK, Caspase inhibitor.

(Figure 5E, 5F). When treated with caspase inhibitor Z-VAD-FMK, the expression of DIABLO was significantly decreased (Figure 5G).

\section{Discussion}

Despite rapid developments in treatment during the past decade, NSCLC continues to be the leading cause 
of tumor-related deaths worldwide (17). Management of patients diagnosed with NSCLC includes surgery, chemotherapy, and radiation therapy. Current tumor targeting therapies have demonstrated excellent efficacy in NSCLC, but drug toxicity and resistance tend to limit the use of chemotherapy. Given that, an efficient and lowtoxicity, good toleration and safety therapy for NSCLC is urgently needed. The present study provides in vitro and in vivo evidence that BSE, already used in traditional Chinese medicine, may be effective as chemotherapy against NSCLC because it can inhibit cell proliferation and induce apoptosis of tumor cells. We have also shown that these anti-tumor effects may involve up-regulation of the apoptosis regulator DIABLO.

BSE has been used as a traditional Chinese medicine for their therapeutic properties in lung diseases for centuries. The present work extends our previous study, in which we found that BSE can promote pulmonary vascular remodeling and mitigate pulmonary inflammation and further relieve pulmonary arterial hypertension (18). In the present study, we found that an ethanolic extract from the same plant can significantly reduce the volume of NSCLC xenografts. This finding, together with centuries' long experience of using this BSE extract in traditional medicine, means that it may be more tolerable to patients than other chemotherapy drugs. These advantages are very important in the therapy of advanced NSCLC patients. Our results in vitro and in vivo suggest that BSE could be an effective treatment strategy in NSCLC.

Our finding that DIABLO helps mediate the anti-tumor effects of BSE is consistent with previous work showing a significant association between higher DIABLO expression in NSCLC tissue and longer tumor-free and overall survival (19). Further research is needed to clarify the details of how the DIABLO protein exerts anti-tumor effects. In addition, other DEGs that we identified in the NSCLC cells have also been linked to cell apoptosis, which is regulated by DIABLO. For example, $A N K 2$ encodes ankyrin, which plays a crucial role in cell growth, membrane transportation, and metastasis of cancer cells (20). By downregulating $A N K 2$, miR-647 can accelerate the druginduced apoptosis of gastric cancer cells (21).

In conclusion, we present in vitro and in vivo evidence that BSE inhibits NSCLC cell proliferation and induces apoptosis, and these effects appear to involve the up-regulation of DIABLO. We will further identify the mechanism by which DIABLO was regulated in NSCLC and biological process involved on DIABLO expression is up- or down-regulated by
BSE. Our findings provide the foundation for future efforts to discover improved therapies against NSCLC.

\section{Acknowledgments}

The authors thank Dr. Qinjie Weng and Dr. Xiaoyang Dai, Zhejiang Province Key Laboratory of Anti-Cancer Drug Research, College of Pharmaceutical Sciences, Zhejiang University, China, for xenograft-model building.

Funding: This work was supported by the National Natural Science Foundation of China (81903898 to WXX); Zhejiang Provincial Natural Science Foundation of China, Zhejiang Province, People's Republic of China (LYQ20H310001 to YJL, LY20H310001 to YWZ, and LQ19H280001 to HYD); the Medical and Health Research Program of Zhejiang Province (2018KY297 to YJL, 2019KY047 to JS, and 2019KY559 to LL); Chinese Medicine Research Program of Zhejiang Province (2018ZZ006 to PH, 2020ZZ003 to PH, 2021ZZ001 to YWZ); Zhejiang Provincial Program for the Cultivation of New Heath Talents (to YWZ); the Zhejiang Provincial Program for the Cultivation of 151 Talents (to PH); and the Zhejiang Provincial Program for the Cultivation of High-Level Innovative Heath Talents (to PH).

\section{Footnote}

Reporting Checklist: The authors have completed the ARRIVE reporting checklist. Available at https://dx.doi. org/10.21037/atm-21-5409

Data Sharing Statement: Available at https://dx.doi. org/10.21037/atm-21-5409

Conflicts of Interest: All authors have completed the ICMJE uniform disclosure form (available at https://dx.doi. org/10.21037/atm-21-5409). The authors have no conflicts of interest to declare.

Ethical Statement: The authors are accountable for all aspects of the work in ensuring that questions related to the accuracy or integrity of any part of the work are appropriately investigated and resolved. Animal experiments were approved by the Animal Experimental Ethics Committee of the Center for Drug Safety Evaluation and Research of Zhejiang University (ZJU) (No. IACUC-17-163) and were in compliance with the guidelines of Institution Animal Care and Use Committee of Center for Drug Safety Evaluation and Research (CDSER) of Zhejiang University (ZJU) for the 
care and use of animals.

Open Access Statement: This is an Open Access article distributed in accordance with the Creative Commons Attribution-NonCommercial-NoDerivs 4.0 International License (CC BY-NC-ND 4.0), which permits the noncommercial replication and distribution of the article with the strict proviso that no changes or edits are made and the original work is properly cited (including links to both the formal publication through the relevant DOI and the license). See: https://creativecommons.org/licenses/by-nc-nd/4.0/.

\section{References}

1. Siegel RL, Miller KD, Jemal A. Cancer statistics, 2019. CA Cancer J Clin 2019;69:7-34.

2. Almodovar T, Teixeira E, Barroso A, et al. Elderly patients with advanced NSCLC: The value of geriatric evaluation and the feasibility of CGA alternatives in predicting chemotherapy toxicity. Pulmonology 2019;25:40-50.

3. Liang J, Lu T, Chen Z, et al. Mechanisms of resistance to pemetrexed in non-small cell lung cancer. Transl Lung Cancer Res 2019;8:1107-18.

4. Wang SM, Ruan JS, Zhuang J. Small Chunhua oral mouse model of chronic bronchitis and pathological forms of influence and expectorant effects. Fujian Chinese Medicine 2001;32:18-9.

5. Nakajima M. From the Viewpoint of Drug Metabolism Research. Yakugaku Zasshi 2017;137:697-705.

6. Ruan JS. Recent progress on research of Botrychium schaffneri Underw. and components. J China Pharm Univ 2002;33:328-9.

7. Zhuan J, Ruan JS. Study on the effect of Botrychium schaffneri Underw.pill on immune function of mice. Fujian J Trad Chin Med 2007;38:40-1.

8. Yuan Y, Yang B, Ye Z, et al. Sceptridium ternatum extract exerts antiasthmatic effects by regulating Th1/Th2 balance and the expression levels of leukotriene receptors in a mouse asthma model. J Ethnopharmacol 2013;149:701-6.

9. Srinivasula SM, Hegde R, Saleh A, et al. A conserved XIAPinteraction motif in caspase-9 and Smac/DIABLO regulates caspase activity and apoptosis. Nature 2001;410:112-6.

10. Lv Z, Song X, Xu J, et al. The modulation of Smac/ DIABLO on mitochondrial apoptosis induced by LPS in Crassostrea gigas. Fish Shellfish Immunol 2019;84:587-98.

11. Greer RM, Peyton M, Larsen JE, et al. SMAC mimetic (JP1201) sensitizes non-small cell lung cancers to multiple chemotherapy agents in an IAP-dependent but TNF- $\alpha$ - independent manner. Cancer Res 2011;71:7640-8.

12. Chou TC. Theoretical basis, experimental design, and computerized simulation of synergism and antagonism in drug combination studies. Pharmacol Rev 2006;58:621-81.

13. Ritchie ME, Phipson B, Wu D, et al. limma powers differential expression analyses for RNA-sequencing and microarray studies. Nucleic Acids Res 2015;43:e47.

14. Lu P, Weaver VM, Werb Z. The extracellular matrix: a dynamic niche in cancer progression. J Cell Biol 2012;196:395-406.

15. Reimand J, Kull M, Peterson H, et al. g:Profiler-a web-based toolset for functional profiling of gene lists from large-scale experiments. Nucleic Acids Res 2007;35:W193-200.

16. Subramanian A, Kuehn H, Gould J, et al. GSEA-P: a desktop application for Gene Set Enrichment Analysis. Bioinformatics 2007;23:3251-3.

17. Romine PE, Martins RG, Eaton KD, et al. Long term follow-up of neoadjuvant chemotherapy for non-small cell lung cancer (NSCLC) investigating early positron emission tomography (PET) scan as a predictor of outcome. BMC Cancer 2019;19:70.

18. Xin WX, Li QL, Fang L, et al. Preventive Effect and Mechanism of Ethyl Acetate Extract of Sceptridium ternatum in Monocrotaline-Induced Pulmonary Arterial Hypertension. Chin J Integr Med 2020;26:205-11.

19. Dai CH, Li J, Shi SB, et al. Survivin and Smac gene expressions but not livin are predictors of prognosis in non-small cell lung cancer patients treated with adjuvant chemotherapy following surgery. Jpn J Clin Oncol 2010;40:327-35.

20. Zhu D, Bourguignon LY. Interaction between CD44 and the repeat domain of ankyrin promotes hyaluronic acidmediated ovarian tumor cell migration. J Cell Physiol 2000;183:182-95.

21. Cao W, Wei W, Zhan Z, et al. Regulation of drug resistance and metastasis of gastric cancer cells via the microRNA647ANK2 axis. Int J Mol Med 2018;41:1958-66.

(English Language Editor: J. Jones)

Cite this article as: Liu YJ, Zhang YW, Zheng SL, Zheng XC, Ding HY, Xin WX, Sun J, Li L, Huang P. Botrychium schaffneri Underw. extract acts via DIABLO to induce apoptosis and inhibit proliferation of non-small cell lung carcinoma in vitro and in vivo. Ann Transl Med 2021;9(22):1676. doi: 10.21037/atm-21-5409 


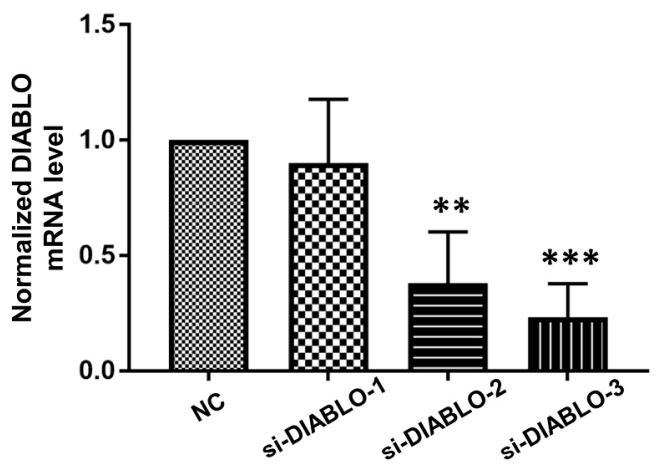

Figure S1 DIABLO levels in NCI-H1299 cultures after transfection with 1 of 3 short interfering RNAs against DIABLO or a negative control siRNA (si-NC). ${ }^{* *}, \mathrm{P}<0.01 ;{ }^{* * *}, \mathrm{P}<0.001$. DIABLO, direct IAP Binding protein with low PI; siRNAs, short interfering RNAs. 